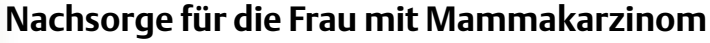 \\ „To follow-up or not to follow-up“
}

\author{
H. Kreis, S.U. Malur, M.W. Beckmann \\ Universitäts-Brustzentrum Franken, Frauenklinik, Universitätsklinikum Erlangen \\ (Direktor: Prof. Dr. M.W. Beckmann)
}

klinikarzt 2004; 33 (11): 331-334

K rebserkrankungen sind schicksalhafte Einschnitte im Leben einer Frau mit zumeist weit reichenden Veränderungen - sowohl für die Betroffenen selbst als auch für Personen ihrer direkten Umgebung. Die individuelle Lebensplanung, zuvor gefasste Lebensentscheidungen und der Sinn des Lebens werden infrage gestellt, die $\mathrm{Zu}$ kunft als unsicher und nicht mehr berechenbar empfunden.

Der Verlauf der Karzinomerkrankung gliedert sich in fünf Phasen (Tab. 1), die sich teilweise überlappen. Die Zeitdauer dieser einzelnen Phasen ist sehr variabel und hängt von verschiedensten Faktoren $a b$, insbesondere aber vom Alter, dem primären Tumorstadium und der primären Behandlung. Die Anzahl der Frauen, die sich in den einzelnen Phasen der Karzinomerkrankung befinden, ist sehr unterschiedlich, wobei sich der höchste Anteil der Frauen in der Phase III, der Phase der Nachsorge und Rehabilitation, befindet.

Exakte Informationen über die Anzahl der primär erkrankten Frauen, der Frauen in aktueller Therapie oder der Frauen mit Metastasen/Rezidiv bzw. der karzinombedingten Todesfälle stehen nicht zur Verfügung. Für gesundheitspolitische Kosten-Nutzen-Analysen sind

Das strukturierte Nachsorge-Konzept nach einer potenziell kurativen Primärtherapie ist klinisch orientiert. Mit einem informativen Aufklärungsgespräch, einer ausführlichen Anamneseerhebung und einer klinischen, symptomorientierten Untersuchung ist es individuell ausgerichtet. Nur bei klinischem Hinweis, Symptomen oder zum Nachweis von Lokalrezidiven ist es notwendig, eine apparative Diagnostik einzusetzen. In den Vordergrund tritt vielmehr die „sprechende Medizin“ mit persönlicher Betreuung der Betroffenen. Die Tumornachsorge gliedert sich daher in einen allgemeinen und einen speziellen Teil, wobei Letzterer organspezifische Symptome, Untersuchungsfrequenzen und Inhalte umfasst.

diese Zahlen aber unabdingbare Vorraussetzungen. Basierend auf den Informationen aus verschiedenen Krebsregistern lebten in Deutschland im Jahr 2003 schätzungsweise 360000 Frauen in der Lebensphase nach primärem Mammakarzinom, teilweise mit adjuvanter Hormontherapie. 55000 Frauen waren an einem MammakarzinomRezidiv oder an -Metastasen erkrankt.

\section{Unklare Datenlage}

Während die Datenlage bezüglich Diagnose und Therapie von gynäkologischen Malignomersterkrankungen als zufrieden stellend gelten kann und prospektiv randomisierte klinische Studien bezüglich der Rezidivtherapie verfügbar sind, ist die Strategie für die Zeit dazwischen - also die Zeit nach der Primärtherapie, das so genannte „Follow up“ bzw. die Nachsorge - bis heute noch nicht standardisiert (1).
In einer Zeit, in der in der Medizin einerseits der Kostendruck wächst und die Integration von Wissen der evidenzbasierten Medizin („evidence based medicine“; EBM) mit Recht eingefordert wird, steht auf der anderen Seite die Patientin mit ihren subjektiven Ängsten und Wünschen. Zurzeit gibt es weder einen „systematic review“", noch eine „Meta-Analyse“ oder eine prospektiv-randomisierte Studie, die einen Vorteil bezüglich eines verlängerten Überlebens von Malignompatientinnen bei intensiver Nachsorge beweist! Doch die evidenzbasierte Medizin ist per definitionem nicht ein starres Festhalten an Daten, sondern vielmehr die Integration der aktuellen Datenlage in die eigenen klinischen Erfahrungen, wobei zugleich die Wünsche der Patientinnen berücksichtigt werden müssen. So erscheint die Entscheidung, die Patientin nachzubeobachten - also „to follow-up“ - trotz der aktuellen Datenlage nur allzu verständlich zu sein. 


\section{Tab. 1 Fünf Phasen der Karzinom- erkrankung}

Phase I von der Diagnose zur Therapieplanung

Phase II Durchführung der Therapie

Phase III onkologische Nachsorge/Rehabilitation

Phase IV Rezidiv/Metastasen

Phase V Palliation/Lebensende

Die Zusammenstellung von Nachsorgeschemata stößt aufgrund der Abwesenheiten eindeutiger Evidenz auf Schwierigkeiten und ist dementsprechend zu einem Großteil auf Expertenmeinungen und auf den Konsensus von Fachgesellschaften in Form von Leit- oder Richtlinien angewiesen. Noch Anfang der 90er Jahre war die Nachsorge nach einem starren, apparativen und technisch orientierten Schema ausgerichtet. Doch Daten einiger prospektiv-randomisierter Studien belegten, dass die Effektivität dieser Untersuchungsschemata und -methoden limitiert ist. Allgemein wird angenommen, dass die Früherkennung eines möglichen Rezidivs die Behandlung erleichtert und somit zu besseren Ergebnissen für die Patientin führt. Dies ist allgemein nicht bewiesen und für die einzel- nen gynäkologischen Tumorentitäten differenziert zu betrachten.

\section{Wie sollte die Nachsorge aussehen?}

Eine individuell ausgerichtete, risiko- und erkrankungsadaptierte Nachsorge sollte - falls möglich immer von ein und demselben Arzt durchgeführt werden, um ein Vertrauensverhältnis aufzubauen und um die Wünsche und Prioritäten der Patientin längerfristig kennen zu lernen (Überlebenszeit versus Lebensqualität). Dazu muss grundsätzlich immer ein informatives und persönliches Informationsgespräch und eine ausführliche und gezielte Anamneseerhebung zählen. Beides sollte individuell auf die Patientin und auf die Malignomentität ausgerichtet und klinisch-symptomorientiert sein und nur eingeschränkt apparative Diagnostik beinhalten. Eine gute Dokumentation (z.B. in Form eines Nachsorgepasses) sollte sowohl zum Nutzen der Patientin wie auch des Arztes selbstverständlich sein. Auch das heute in der Medizin immer häufiger diskutierte Prinzip „So wenig wie möglich, aber so viel wie nötig" sollte bei der Nachsorge berücksichtigt werden.
Als Konsequenz aus den wenigen prospektiven Nachsorgestudien und den aktuellen Einflussfaktoren hat sich ein klinisch orientiertes strukturiertes Nachsorgekonzept nach potenziell kurativer Primärtherapie entwickelt (Abb. 1). Im Vordergrund steht die individuelle Ausrichtung mit einem informativen Gespräch, einer ausführlichen, strukturierten Erhebung der Anamnese und einer klinischen, symptomorientierten Untersuchung. Nur bei klinischem Hinweis oder Symptomen ist es notwendig, die apparative Diagnostik einzusetzen. Stärker in den Vordergrund tritt der Begriff der „sprechenden Medizin“ mit persönlicher Betreuung, dies wird als Aspekt der professionellen ärztlichen Tätigkeit anerkannt. Denn besonders wichtig bei der Nachsorge sind die Bedürfnisse der Patientin. Daraus ergibt sich die Handlungsmaxime mit einer Verbesserung der Heilungschancen oder der Lebensverlängerung und/oder Verbesserung bzw. Erhaltung der Lebensqualität.

\section{„Allgemeine“ Tumornachsorge}

Die Tumornachsorge gliedert sich in einen „allgemeinen Teil“, der die Betreuung der Patientinnen und

\section{Abb. 1 Strukturiertes Nachsorgekonzept}

allgemeine Maßnahmen

Übelkeit, Erbrechen

chronisches Fatigue-Syndrom

Schmerzen

Narben

Lymphödem/sekundäre Folgen

körperliche Leistungsfähigkeit

Hormonausfallserscheinungen

Sensibilitätsstörung

Lokalrezidiv

Metastasen

Zweiterkrankung/-malignom
Therapiemonitoring

Langzeittherapien

Nebenwirkungen

Langzeitfolgen

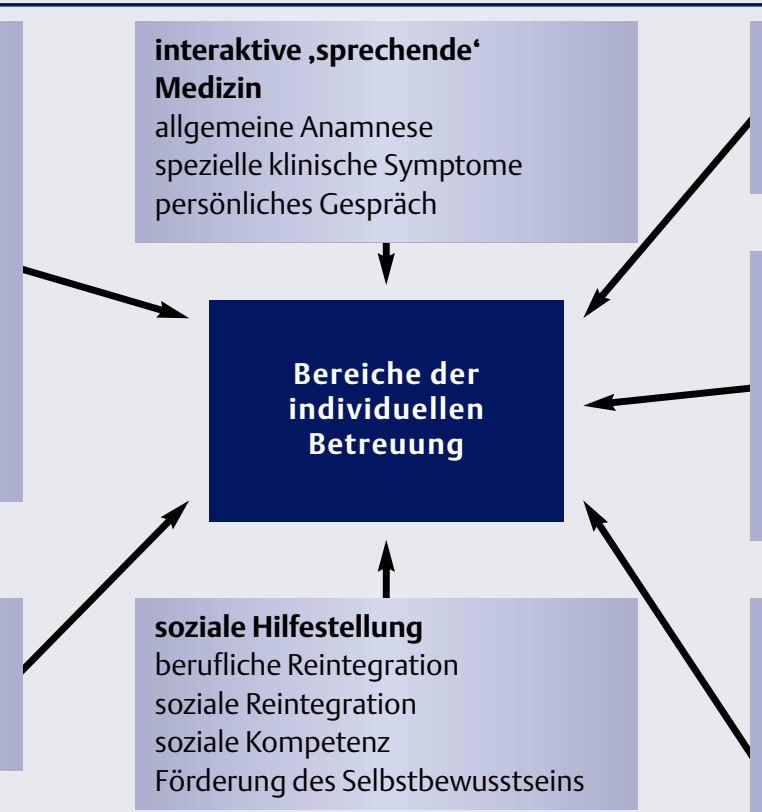

Förderung des Selbstbewusstseins spezielle Maßnahmen

Kompensation/Selbstversorgung

berufsbezogene Trainingsaktivitäten

Prothetik

psychologische Intervention depressive Verstimmung, Ängste, Nervosität, Anspannung Stärkung des Selbstwertgefühls Krankheitsbewältigung Familie, Partnerschaft, Sexualität Todesangst

Schulung und Information

Ernährung und Lebensstil

Risikoreduktion

Prophylaxe von Lymphödem,

Thrombose, Kontrakturen

Stressbewältigung

komplementäre Therapien

familiäre Disposition 


\section{Tab. 2 Nachsorgeschema „Mammakarzinom“}

\section{Anamnese (allgemein und tumorspezifisch)}

vor allem Arm- oder Knochenschmerzen, Entwicklung eines Lymphödems des Arms, lokale Rötung oder Knotenbildung, Atembeschwerden, Husten, Gewichtsverlust, allgemeine Abgeschlagenheit, gastrointestinale/epigastrische Beschwerden,

Therapiespätfolgen (Abduktionshemmung des Armes, Strahlendermatitis, klimakterische Beschwerden vor allem bei jungen Frauen, Osteoporose, kardiale Symptome, Hirnleistungsstörung und anderes)

Besonderheiten: zum Beispiel lokoregionäres Rezidiv, kontralaterales Mammakarzinom

\section{Selbstuntersuchung der Mamma}

lokal (Operationsgebiet), axillär

\section{klinische Untersuchung}

Inspektion und Palpation der lokalen und lokoregionären Situation und der kontralateralen Brust

\section{gynäkologische Untersuchung}

Spekulumeinstellung, bimanuelle rektovaginale Untersuchung, Entnahme von Zervixzytologieabstrichen (PAP), Untersuchung der inguinalen Lymphknoten beidseits, Vaginalultraschall

\section{Mammadiagnostik}

Mammografie, Mammasonografie, eventuell MRT

\section{Tumormarker}

CEA (karzinoembryonales Antigen) und CA 15-3 (Krebsantigen 15-3) im Serum (Kontrollen nur, falls bei Primärdiagnose erhöht oder Verdacht auf Rezidiv)

\section{sonstige bildgebende Untersuchungen}

Thoraxröntgen, CT, MRT, Ultraschall (Oberbauch), Skelettszintigrafie

\begin{tabular}{llll}
$\begin{array}{l}\text { Zeitplan } \\
\text { Untersuchung }\end{array}$ & $\begin{array}{l}\mathbf{1 . - 3 . ~ J a h r} \\
\text { (Kontrollen: dreimonatlich) }\end{array}$ & $\begin{array}{l}\text { 4.-5. Jahr } \\
\text { (Kontrollen: sechsmonatlich) }\end{array}$ & $\begin{array}{l}\mathbf{6 . - 1 0 . ~ J a h r ~} \\
\text { (Kontrollen: jährlich) }\end{array}$ \\
\hline • Anamnese & bei jeder Kontrolle & bei jeder Kontrolle & bei jeder Kontrolle \\
\hline - Selbstuntersuchung & monatlich & monatlich & monatlich \\
\hline - klinische Untersuchung der Mamma & bei jeder Kontrolle & bei jeder Kontrolle & jährlich \\
\hline - gynäkologische Untersuchung & jährlich & jährlich & jährlich \\
\hline - Mammadiagnostik & & & \\
\hline - ipsilaterale Brust & mindestens jährlich & jährlich & jährlich \\
\hline - kontralaterale Brust & jährlich & jährlich & jährlich \\
\hline - Tumormarker & nicht in der Routine & nicht in der Routine & nicht in der Routine \\
\hline - sonstige Bildgebung & bei klinischem Verdacht & bei klinischem Verdacht & bei klinischem Verdacht
\end{tabular}

ihre Information bezüglich Übelkeit und Erbrechen, dem chronischen Fatigue-Syndrom, Schmerzen, Narben, Lymphödem mit den sekundären Folgen, Hormonausfallserscheinungen, Sensibilitätsstörungen und Lokalrezidiv, Metastasen oder Zweiterkrankungen/-malignom umfasst. Schulung und Information betreffen Ernährung und Lebensstil, die Möglichkeiten der Risikoreduktion, der Prophylaxe von Lymphödemen, Stressbewältigung sowie die Beratung über komplementäre Therapien und die familiäre Disposition.

Soziale Hilfestellungen sollten die berufliche und soziale Reintegration und die Förderung der sozialen Kom- petenz und des Selbstbewusstseins umfassen. Zentraler Punkt der an den Patientinnen orientierten gynäkologisch-onkologischen Nachsorge ist jedoch die psychologische Intervention. Hierzu zählt die Betreuung der Betroffenen bei depressiver Verstimmung, Ängsten, Nervosität oder Anspannung. Dieses kann durch psychologisch-psychotherapeutische Interventionen mit einer Stärkung des Selbstwertgefühls und dem Versuch der Krankheitsbewältigung erfolgen. Auch Aspekte der Familie einschließlich Partnerschaft und Sexualität sollten drin einbezogen werden. Zentrales Thema ist die Bewältigung der Lebens- und der Todesangst.

\section{„Spezielle“ Tumornachsorge}

Der „spezielle Teil“ der Nachsorge umfasst die spezifischen Symptome der Patientin, die Untersuchungsfrequenz und die Untersuchungsinhalte. Zusätzlich wird die gesetzliche Krebsfrüherkennung integriert, da für einige Karzinomarten ein erhöhtes Zweitkarzinomrisiko besteht.

Das hier vorgestellte Nachsorgeschema für das Mammakarzinom ist eine Zusammenstellung der Arbeitsgemeinschaft Gynäkologische Onkologie (AGO) sowie erarbeiteten Leitlinien und Bestandteil der derzeit publizierten Leitlinien der Deutschen Krebsgesellschaft (DKG) bzw. 
der Deutschen Gesellschaft für Gynäkologie und Geburtshilfe (DGGG) (Tab. 2).

\section{Langzeitfolgen \\ der Primärtherapie}

Ziel der Nachsorge ist zudem auch die Erkennung längerfristiger Therapie- und Krankheitsfolgen. In der Nachsorge des Mammakarzinoms seien einige besonders erwähnt:

- Kardiotoxizität der Anthrazykline

- gonadale Toxizität

- Arthralgien bei Gabe des Aromatasehemmers Anastrozol

- tumorbedingtes Fatigue-Syndrom

- Lymphödem.

Mit steigender Zahl an Patientinnen, die im Rahmen eines neoadjuvanten oder adjuvanten Therapieregimes mit Anthrazyklinen behandelt werden, werden auch immer mehr Patientinnen mögliche kardiotoxische Nebenwirkungen erleben. Häufig ist dabei die Entwicklung einer akuten oder chronischen Kardiomyopathie mit konsekutiver Herzinsuffizienz. Unterschieden wird hier zwischen

- einer akuten oder subakuten dosisunabhängigen Frühform

- einer chronischen Form, die innerhalb eines Jahres nach Beginn der Anthrazyklinbehandlung auftritt und

- der „Late-onset“-Kardiotoxizität, welche Patientinnen betrifft, die eine Anthrazyklintherapie erhalten haben, die mehr als ein Jahr zurückliegt (3).

Die beiden letztgenannten Formen können in den Zeitraum der Nachsorge fallen. Somit müssen die betroffenen Patientinnen - abhängig von dem Krankheitsstadium - einer adäquaten kardiologischen Therapie zugeführt werden.

Im Rahmen einer zytostatischen Chemotherapie kann es bei prämenopausalen Patientinnen durch die toxische Wirkung der Behandlung auf die Ovarien zu einer vorzeitigen Induktion der Menopause kommen (4). Die damit verbundenen Osteoporoserisiken und das klimakteri- sche Syndrom bedürfen der Beratung und einer Therapieempfehlung. Insbesondere bei prämenopausalen Frauen sind diese Punkte wichtige Bestandteile in der Nachsorge des Mammakarzinoms.

Arthralgien bei Einnahme des Aromatasehemmers Anastrozol sind eine häufig geäußerte Nebenwirkung. Auch hier sind neben der symptomatischen Therapie mit nichtsteroidalen Antiphlogistika das Gespräch und die Aufklärung wichtig.

Das tumorbedingte Fatigue-Syndrom kann auch nach erfolgreicher Therapie ein bleibendes Problem sein. Es handelt sich um ein unspezifisches und kaum objektivierbares Syndrom einer allgemeinen Kraftlosigkeit, das die Lebensqualität erheblich beeinflussen kann. Die Genese des Fatigue-Syndroms ist in ihrer Gesamtheit nicht geklärt, entsprechend schwierig gestaltet sich seine Therapie. Neben einer symptomatischen Therapie (Weckamine, Antidepressiva oder Kortisonpräparate, intermittierende Bluttransfusionen oder Gabe von Erythropoetin) sind Information, Aufklärung und Dialog die wichtigste Hilfe für die Patientin, um so die Krebsdiagnose und ihre Folgen - insbesondere auch das Fatigue-Syndrom - bewältigen zu können.

Das Armödem kann primär oder sekundär entstehen. Nach Ausschluss eines Rezidives ist eine eingehende Beratung über Verhaltensmaßnahmen notwendig und je nach Ausprägung die Einleitung von physikalischen Therapien (z.B. Kompressionsstrumpf, Lymphdrainage oder Krankengymnastik) zu empfehlen.

Follow-up Care for Women with Breast Cancer - „To follow up or not to follow up“

The structural programme of followup care for potentially cured women in the adjuvant setting is clinically oriented. It encompasses individualisation of care with an informative, detailed and structured talk and a clinically directed exam. Only the detection of clinical signs and symptoms leads to further diagnostic procedures. 'Talking Care' is the way of personal care for the diseased women. Follow-up care is divided into a general and a specific part. The latter summarizes organ-specific symptoms, frequencies and nature of exams.

\section{Key Words}

follow up - dialogue - physical examination - diagnostic investigation - prolonged toxicity - quality of live

\section{Literatur bei den Verfassern}

Anschrift für die Verfasser

Dr. Hilde Kreis Frauenklinik Universitätsklinikum Erlangen Universitätsstr. 21-23 91054 Erlangen 